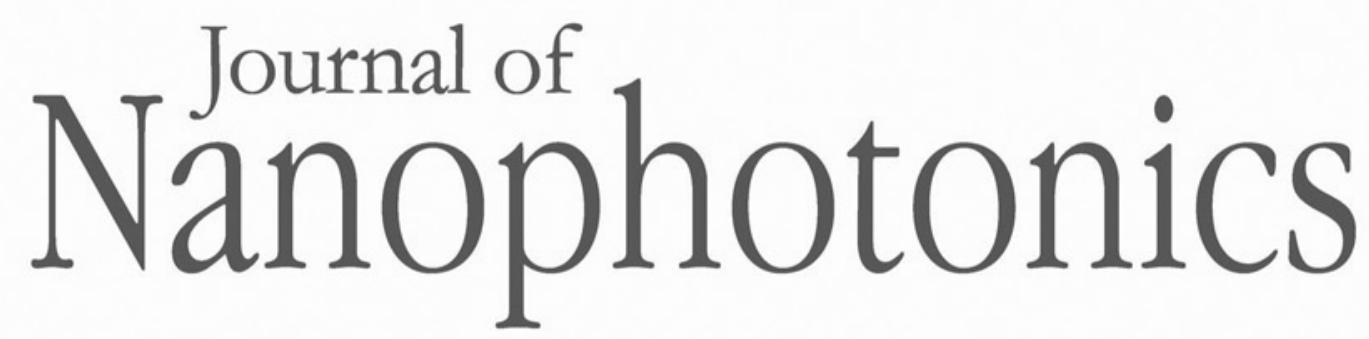

\title{
Excitation of quantum dot by femtosecond plasmon-polariton pulse focused by conducting cone
}

Pavel A. Golovinski

Valeri A. Astapenko

Egor S. Manuylovich

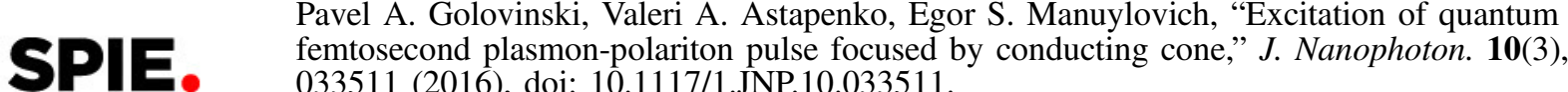




\title{
Excitation of quantum dot by femtosecond plasmon-polariton pulse focused by conducting cone
}

\author{
Pavel A. Golovinski, ${ }^{a}$ Valeri A. Astapenko, ${ }^{b}$ and Egor S. Manuylovich ${ }^{\mathrm{b}, *}$ \\ ${ }^{a}$ Voronezh State Architecture and Engineering University, Physical Research Laboratory, \\ Dvattsatiletiya Oktyabrya, 84, Voronezh 394030, Russia \\ ${ }^{b}$ Moscow Institute of Physics and Technology, Institutsky Side Street 9, \\ Dolgoprudny 141700, Russia
}

\begin{abstract}
A surface plasmon polariton is an electromagnetic wave that propagates along an interface between two materials with dielectric permittivity of opposite signs. Such waves can be focused by metal waveguides of special geometry. The spatial distribution for a nearfield strongly depends on a linear chirp of the laser pulse, which can partially compensate the wave dispersion. Field distribution is calculated for different chirp values, opening angles, and distances. The spatial selectivity of excitation of quantum dots using focused fields is shown using Bloch equations. (C) 2016 Society of Photo-Optical Instrumentation Engineers (SPIE) [DOI: 10 .1117/1.JNP.10.033511]
\end{abstract}

Keywords: plasmon polariton; superfocusing; femtosecond pulse; quantum dot; excitation.

Paper 15192SS received Dec. 21, 2015; accepted for publication Mar. 23, 2016; published online Apr. 18, 2016.

\section{Introduction}

Generation of femtosecond pulses of variable shape enables control of complex quantum systems. ${ }^{1,2}$ One of the main problems in nano-optics is the focusing of electromagnetic fields to the nanoscale regions. ${ }^{3}$ This is especially important if closely spaced nano-objects should be controlled separately. ${ }^{4}$

Evanescent fields play a central role in nano-optics. ${ }^{5}$ They are characterized by the fact that the strength of the evanescent electromagnetic field decreases exponentially with increasing distance from the interface. A surface plasmon polariton (SPP) meets this condition. An SPP is a quasiparticle that propagates along an interface between two media (metal and dielectric) and represents oscillations of the conduction electrons in the metal coupled with the oscillations of the electromagnetic field in the dielectric. Near-field optics related to surface waves is the study in the area of plasmonics or nanoplasmonics. ${ }^{6}$

The combination of adaptive control and the unique properties of SPPs allows to dynamically localize field at subwavelength nanoscale levels. SPPs are convenient for subwavelength spatial control of even broadband optical fields. ${ }^{7}$ One of the most prominent approaches to spacetemporal electromagnetic field superfocusing is related to using SPP propagation in sharply tapered metal nanorods. ${ }^{8}$ In theoretical works, ${ }^{9,10}$ the ability to generate SPP wave packets in nanowires has been shown. These packets propagate along the nanowires and represent a superposition of plasmon modes in multiresonance systems.

Variation in the effective refractive index with a decrease in the diameter of the tapered waveguide leads to a continuous change in the optical modes and adiabatic focusing of SPP as it approaches the apex of the cone. ${ }^{11}$ It has been experimentally proven that the metal tapered waveguide allows for the focus of SPPs in a region as small as a few tens of nanometers. ${ }^{12,13}$ Femtosecond pulse propagation in conical gold nanoprobes has been experimentally observed. ${ }^{14}$

*Address all correspondence to: Egor S. Manuylovich, E-mail: egor.manuylovich@gmail.com

$1934-2608 / 2016 / \$ 25.00$ (C) 2016 SPIE 
The details of the nanofocusing mechanism are not yet completely understood even under adiabatic conditions, ignoring radiation damping and reflection. This new optical antenna allows the achievement of far- to near-field transformation of light from the microscale to the nanoscale. The aim of this paper is numerical simulation of the excitation probability of quantum dots (QDs) via focused plasmon-polariton pulse near the nanotip.

\section{Theoretical Model}

Investigation of field enhancement near the cone tip leads to the conclusion that the process is typical for symmetric TM $(m=0)$ waves. This type of polarization can be obtained, e.g., by direct focusing of laser radiation with appropriate polarization on the base of the cone. ${ }^{15}$ In the following consideration, we imply the wavepacket propagation. The main difference of this problem from classical diffraction ${ }^{16}$ is explained by the small diameter of the nanowire compared to the depth of the skin effect and frequency dependence of complex dielectric constants for metals at optical wavelengths.

It has been shown that using three-dimensional (3-D) finite-difference time domain analysis, it is possible to selectively confine either the electric or magnetic field at the tip apex. ${ }^{17} \mathrm{We}$ follow the analytical approach ${ }^{18}$ to the solution of the Maxwell equations. It is convenient to choose a spherical system of co-ordinates as shown in Fig. 1 to consider the field near the cone apex. We assume that the magnetic field is directed along the $\varphi$ axis and depends only on the co-ordinates $r$ and $\theta$. This type of polarization can be obtained, e.g., by direct focusing of the laser radiation of radial polarization on the base of the cone. ${ }^{19}$

For radially polarized modes and conical waveguides, there is no azimuthal angle dependence, and the eigenmodes satisfy the wave equation as follows: ${ }^{20}$

$$
\frac{1}{r} \frac{\partial^{2}}{\partial r^{2}}\left(r H_{\varphi}\right)+\frac{1}{r^{2}} \frac{\partial}{\partial \theta}\left(\frac{1}{\sin \theta} \frac{\partial\left(\sin \theta H_{\varphi}\right)}{\partial \theta}\right)=\frac{\varepsilon}{c^{2}} \frac{\partial^{2} H_{\varphi}}{\partial t^{2}} .
$$

Separating variables gives

$$
\begin{gathered}
H_{\varphi}(r, \theta, t)=R(r) \Psi(\theta) \exp (-i \omega t), \\
\frac{\mathrm{d}^{2} \Psi}{\mathrm{d} \theta^{2}}+\frac{\cos \theta}{\sin \theta} \frac{\mathrm{d} \Psi}{\mathrm{d} \theta}-\left(\eta^{2}+\frac{1}{\sin ^{2} \theta}\right) \Psi=0,
\end{gathered}
$$

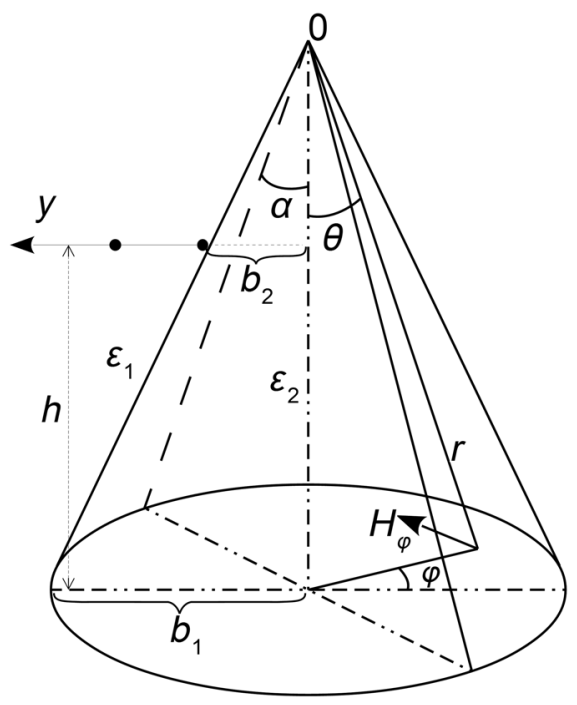

Fig. 1 The geometry of the conical structure. 


$$
\frac{\mathrm{d}^{2} R}{\mathrm{~d} r^{2}}+\frac{2}{r} \frac{\mathrm{d} R}{\mathrm{~d} r}+\left(\frac{\eta^{2}}{r^{2}}+\varepsilon \frac{\omega^{2}}{c^{2}}\right) R=0 .
$$

Assuming the apex angle of the cone $\alpha$ is small, the parameter $\theta \ll 1$. Then the function $\Psi$ obeys the approximate equation

$$
\frac{\mathrm{d}^{2} \Psi}{\mathrm{d} \theta^{2}}+\frac{1}{\theta} \frac{\mathrm{d} \Psi}{\mathrm{d} \theta}-\left(\eta^{2}+\frac{1}{\theta^{2}}\right) \Psi=0
$$

where $x=\eta \theta$. The $\mathrm{TM}_{0}$ solution to Eq. (5) can be expressed in terms of the modified Bessel functions ${ }^{20}$ as

$$
\Psi(x)=A I_{1}(x)+B K_{1}(x) .
$$

This choice of functions is justified by the evanescent nature of this type of waves. Solving the equations of the radial and angular parts gives ${ }^{18}$

$$
H_{\varphi} \sim \frac{A(\omega)}{\sqrt{r}} \Psi(\theta) \exp \left(-i \omega t-i \eta \ln \frac{r}{r_{0}}\right),
$$

where $\eta$ is the separation constant to be determined from the boundary conditions on the surface and distance $r_{0}$ from the apex fixes the wave phase.

Following the Maxwell equations, we have

$$
\begin{gathered}
E_{r}=\frac{i c}{\omega \varepsilon} \frac{1}{r \sin \theta} \frac{\partial\left(H_{\varphi} \sin \theta\right)}{\partial \theta}, \\
E_{\theta}=-\frac{i c}{\omega \varepsilon} \frac{\partial}{\partial r}\left(r H_{\varphi}\right) .
\end{gathered}
$$

The final asymptotic form for electric field components is

$$
\begin{gathered}
E_{r}(\omega)=-\frac{i c}{\omega \varepsilon_{2}} \frac{\eta A(\omega)}{r^{3 / 2}} I_{0}(\eta \theta) \exp \left(-i \eta \ln \frac{r}{r_{0}}\right), \\
E_{\theta}(\omega)=\frac{i c}{\omega \varepsilon_{2}}\left(\eta+\frac{i}{2}\right) \frac{A(\omega)}{r^{3 / 2}} I_{1}(\eta \theta) \exp \left(-i \eta \ln \frac{r}{r_{0}}\right),
\end{gathered}
$$

for $\theta \leq \alpha$ and

$$
\begin{gathered}
E_{r}(\omega)=\frac{i c}{\omega \varepsilon_{1}} \frac{\eta B(\omega)}{r^{3 / 2}} K_{0}(\eta \theta) \exp \left(-i \eta \ln \frac{r}{r_{0}}\right), \\
E_{\theta}(\omega)=\frac{i c}{\omega \varepsilon_{1}}\left(\eta+\frac{i}{2}\right) \frac{B(\omega)}{r^{3 / 2}} K_{1}(\eta \theta) \exp \left(-i \eta \ln \frac{r}{r_{0}}\right),
\end{gathered}
$$

for $\theta \geq \alpha$. The monochromatic mode solutions in the form of Eqs. (10)-(13) are the starting point in the construction of a wave packet.

\section{Plasmon-Polariton Pulse Focusing}

We consider a short pulse of SPPs, propagating in a metallic cone, for initial condition $(r=R$, $\theta=0$ ) having the chirped pulse form

$$
H_{\varphi}(R, \theta, t)=f(t)=f_{0} \exp \left(-a t^{2}-i \omega_{0} t-i \beta t^{2}\right) .
$$

The Fourier transform of this pulse gives 


$$
H_{\varphi}(R, \theta, \omega)=\frac{f_{0}}{\sqrt{4 \pi(a+i \beta)}} \exp \left(-\frac{\left(\omega-\omega_{0}\right)^{2}}{4(a+i \beta)}\right) .
$$

For electric field components, space-time evolution of the wave packet can be expressed as the Fourier integral

$$
E_{j}(r, \theta, t)=\int_{-\infty}^{\infty} \tilde{f}(\omega) E_{j}(\omega) \mathrm{e}^{i \omega t} \mathrm{~d} \omega, \quad j=r, \theta .
$$

The dependence of $E_{j}(\omega)$ on frequency $\omega$ has to be known for numerical calculation of the integral in Eq. (16).

The dispersion relation is determined by the boundary conditions. The continuity conditions on the surface $(\theta=\alpha)$ lead to the equations

$$
\begin{gathered}
\varepsilon_{2}(\omega) \frac{I_{1}[\alpha \eta(\omega)]}{I_{0}[\alpha \eta(\omega)]}=-\varepsilon_{1}(\omega) \frac{K_{1}[\alpha \eta(\omega)]}{K_{0}[\alpha \eta(\omega)]}, \\
\frac{B(\omega)}{A(\omega)}=\frac{I_{1}[\alpha \eta(\omega)]}{I_{0}[\alpha \eta(\omega)]},
\end{gathered}
$$

where dielectric permittivity of metal $\varepsilon_{2}=\varepsilon_{2}^{\prime}+i \varepsilon_{2}^{\prime \prime}$ is taken from Ref. 21. Equation (17) determines the eigenvalue $\eta$ uniquely, while Eq. (18) determines the ratio $A$ and $B$.

The real and imaginary parts of $\eta$ for a silver needle with apex angle $\alpha=0.01$ are plotted in Fig. 2.

By setting $f(t)=H_{\varphi}(R, \theta, t)$, we find $A(\omega)$ and spectral components $E_{r}(r, \omega), E_{\theta}(r, \omega)$ inside the cone; then from Eq. (18), we find $B(\omega)$ and spectral components outside the cone.

Figure 3 shows the results of numerical simulations for the field in the vicinity of the silver nanoneedle for a laser pulse with duration $32 \mathrm{fs}$, carrier wavelength $1550 \mathrm{~nm}$, and positive chirp $\beta=0.013 \mathrm{fs}^{-2}$.

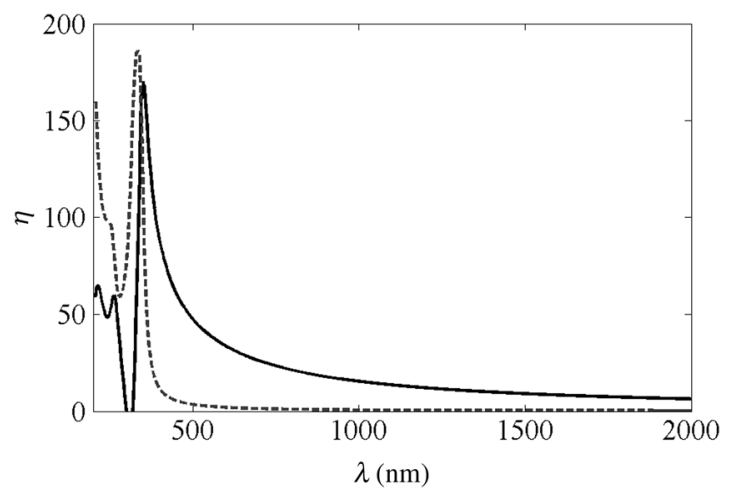

Fig. 2 Dependence of $\operatorname{Re} \eta$ (solid line) and $\operatorname{Im} \eta$ (dotted line) as a function of wavelength $\lambda$.

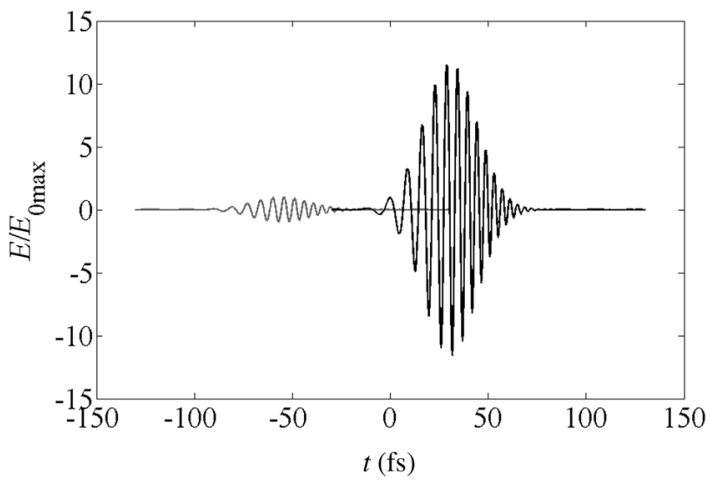

Fig. 3 Compression of SPP wave as a result of propagation starting at the distance $r_{i}=1000 \mathrm{~nm}$ from the apex up to the distance $r_{f}=250 \mathrm{~nm}$. 
The dependence of pulse gain on the cone angle is plotted in Fig. 4 for a silver cone (all other parameters are the same as for Fig. 3 except the cone angle).

\section{Excitation of Quantum Dot}

A pulse evanescent field is convenient for spatially selective excitation of QDs. Some approaches to the consideration of the QDs are formulated in Refs. 22-24. Let us consider an InGaAs QD with radius equal to $5 \mathrm{~nm}$ on the substrate GaAs. ${ }^{25}$ We take the energy structure of the QD according to the parabolic potential model (see Fig. 5), where the energy gap between the ground level of the valence band and the ground level of the conduction band is equal to $E_{\text {gap }}=1.3 \mathrm{eV} .^{25}$

Energy gaps between the 1 and 2 levels in the conduction and valence bands are equal to $\Delta E_{e, h}=\hbar \sqrt{c_{i} / m_{e, h}^{*}}$, where $m_{e, h}^{*}$ is the effective mass of an electron or a hole and $V(x, y, z)=$ $c_{x} x^{2}+c_{y} y^{2}+c_{z} z^{2}$ is the 3-D parabolic potential. For simplicity, we restricted our measurements to a symmetrical QD, i.e., $c_{x}=c_{y}=c_{z}=c$. For such a QD $c=0.036 \mathrm{eV} / \mathrm{nm}^{2}$, $m_{e}^{*}=0.04 m_{0}, m_{h}^{*}=0.45 m_{0},{ }^{25}$ and $\Delta E_{e}=0.26 \mathrm{eV}, \Delta E_{h}=0.078 \mathrm{eV}$. For the pulse duration of $\Delta t_{\mathrm{FWHM}}=33 \mathrm{fs}$, the spectrum width is equal to $\Delta \omega_{\mathrm{FWHM}}=0.167 \mathrm{PHz}$, or in the energy units, $\Delta E_{\mathrm{HWHM}}=\hbar \Delta \omega_{\mathrm{HWHM}}=\left(\hbar \Delta \omega_{\mathrm{FWHM}} / 2\right)=0.055 \mathrm{eV}$. Thus $\Delta E_{\mathrm{HWHM}}<\Delta E_{e, h}$,

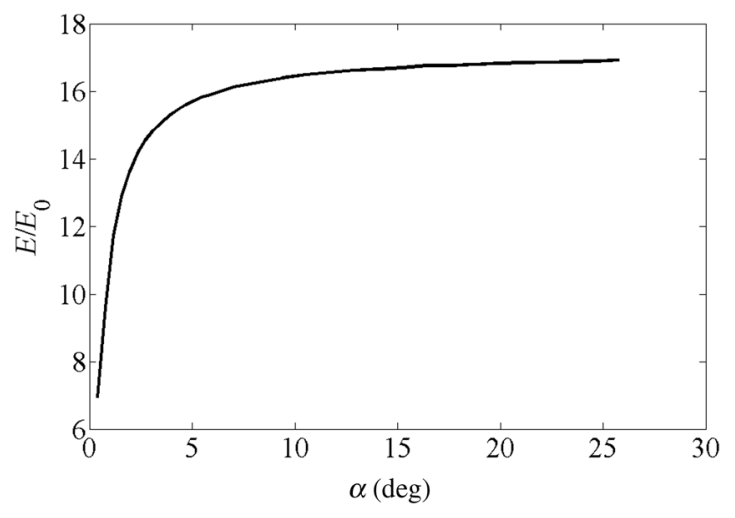

Fig. 4 Pulse gain as a function of a cone angle.

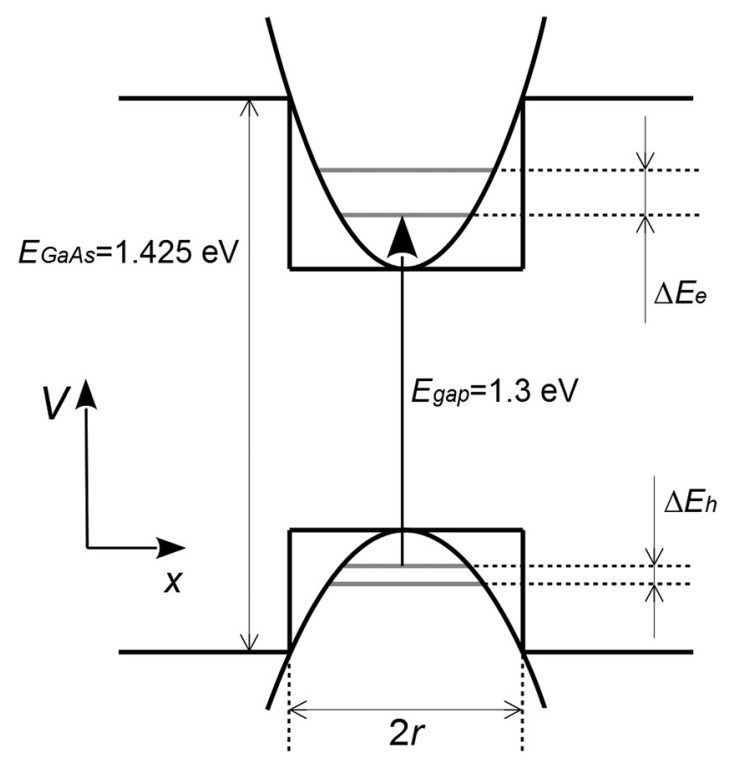

Fig. 5 The energy structure of the QD as the energy structure of an infinite parabolic well. 
and the two-level system model is correct for the description of photoexcitation of transition between the highest valance band level to the lowest conduction band level in the QD.

Following the model of SPP pulse amplification, one can determine an excitation probability for the QD near the cone. The calculation is based on the Bloch equations ${ }^{26,27}$

$$
\cdot R_{1}=\omega_{\mathrm{QD}} R_{2}-\frac{R_{1}}{T_{2}}, \quad \cdot R_{2}=-\omega_{\mathrm{QD}} R_{1}-\frac{R_{2}}{T_{2}}+2 \frac{d_{0} E(t)}{\hbar} R_{3}, \quad \cdot R_{3}=\frac{R_{3}^{(e)}-R_{3}}{T_{1}}-2 \frac{d_{0} E(t)}{\hbar} R_{2},
$$

where $R=\left(R_{1}, R_{2}, R_{3}\right)$ is the optical Bloch vector, $R_{3}^{(e)}$ is the equilibrium value of $R_{3}$, $\omega_{0}=\omega_{\mathrm{QD}}=E_{\text {gap }} / \hbar=1.98 \mathrm{PHz}$, and $d_{0}$ is the electric dipole moment of the transition in the QD. The excitation probability is equivalent to the upper-level population $N_{2}(t)=$ $\left(1-R_{3}\right) / 2$ after the pulse $(t \gg \tau)$. Initial conditions $R_{1}=R_{1}=0, R_{3}=1$ correspond to the unexcited upper state.

Generally speaking, the evolution of the Bloch vector is determined by the values of the longitudinal $T_{1}$ and transverse $T_{2}$ relaxation time constants. The typical values of these constants are larger than a few picoseconds, i.e., much larger than the femtosecond pulse duration; thus, relaxation can be neglected. ${ }^{28}$ The excitation probability was calculated for the QD located outside the cone depending on the distance $y$ from the cone axis (as shown in Fig. 1). The QD is excited by the electromagnetic field of the SPP pulse focused by the silver cone with parameters $\alpha=0.1, b_{1}=100 \mathrm{~nm}$, and $b_{2}=2.5 \mathrm{~nm}$ (see Fig. 6).

The propagation distance is $h=972 \mathrm{~nm}$, and the field gain is $E_{\max }(0,0) / E_{a}=210$. The initial condition for the pulse is taken $E_{a}$ at the point $(0,-h)$. Figure 7 shows the excitation probability of the QD by ultrashort pulse with zero initial chirp for different values of $E_{a}$. Hereafter, a solid line shows the probability of excitation, and a dashed line is the dependence for the maximum electric field in the pulse.

Figure 7(a) demonstrates that the cone with parameter $b_{2}=2.5 \mathrm{~nm}$ allows us to excite a QD with probability $>0.98$ for $y<6 \mathrm{~nm}$ and probability $<0.1$ for $y>15 \mathrm{~nm}$. Figures 7(b) and 7(c) correspond to different initial values of the field $E_{a}$. They show the oscillations of the excitation probability depending on the transverse co-ordinate $y$. For high excitation selectivity, the initial value of the field should be accurately selected.

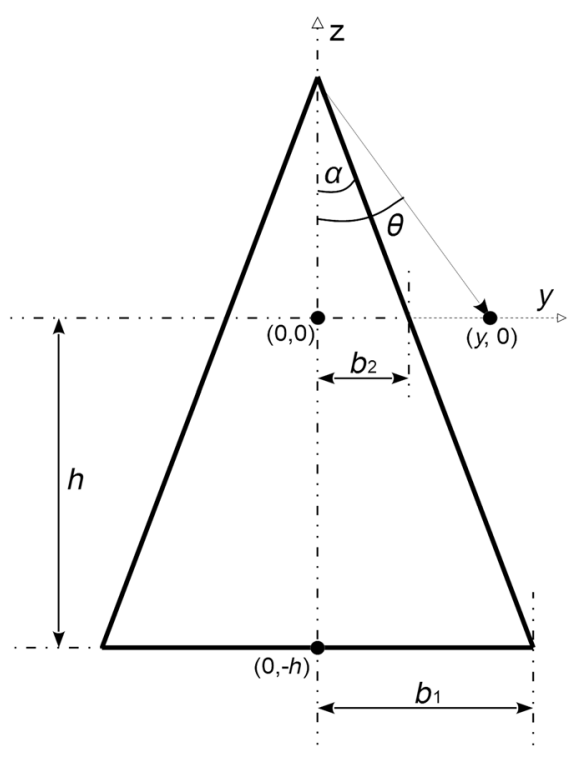

Fig. 6 The relative position of the cone and the QD. The pulse propagates from the point $(0,-h)$ to the point $(0,0)$. The $Q D$ is located at $(y, 0)$. 

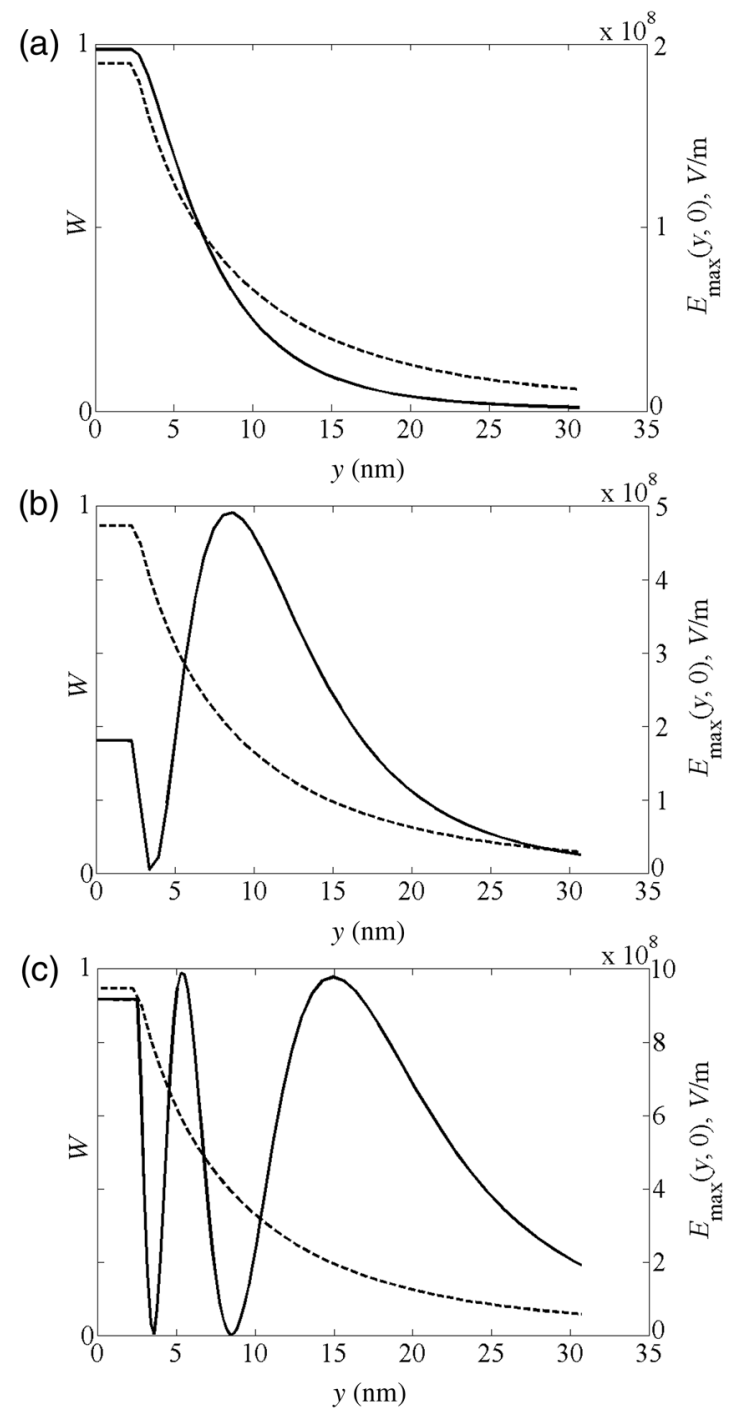

Fig. 7 The probability of excitation of a $Q D$ depending on the distance from the axis of the cone: (a) $E_{a}=10^{6} \mathrm{~V} / \mathrm{m}$, (b) $E_{a}=2.5 \times 10^{6} \mathrm{~V} / \mathrm{m}$, and (c) $E_{a}=5 \times 10^{6} \mathrm{~V} / \mathrm{m}$.

The $y$-dependences of the excitation probability for other pulse parameters (duration and initial chirp) are shown in Figs. 8 and 9.

The results plotted in Fig. 9 lead to the conclusion that nonzero chirp increases the QD excitation probability in the case of frequency detuning.

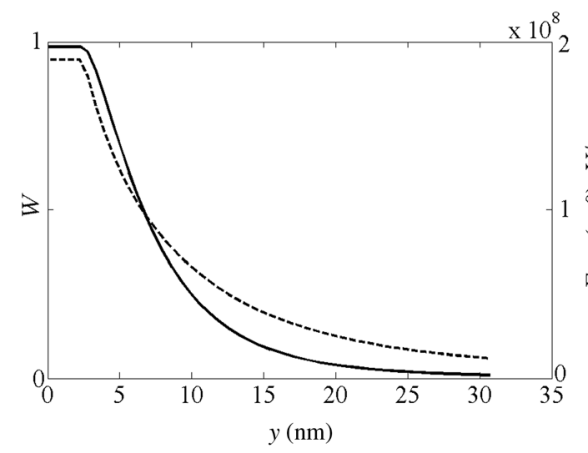

(a)

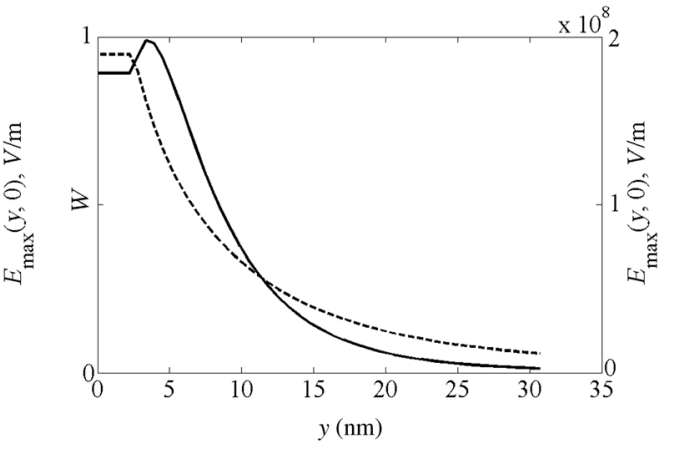

(b)

Fig. 8 The excitation probability of a QD depending on the distance from the axis of the cone. Pulse duration: (a) $\tau=32$ fs and (b) $\tau=42$ fs. 


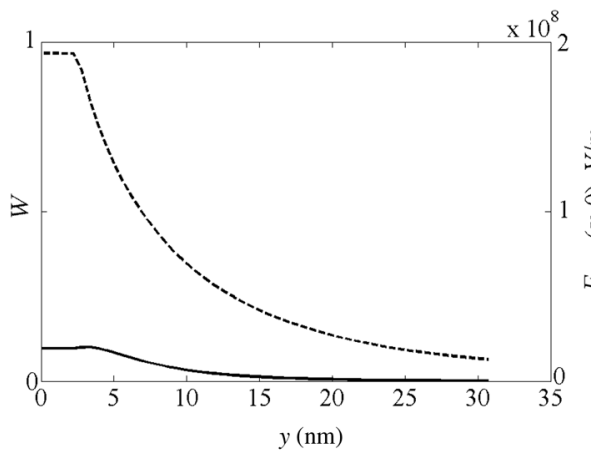

(a)

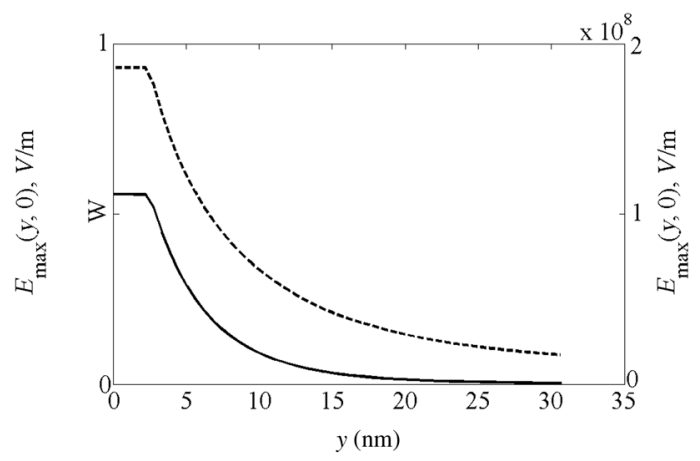

(b)

Fig. 9 The excitation probability of a QD depending on the distance from the axis of the cone. Initial pulse chirp: (a) $\beta=0$ and (b) $\beta=0.062 \mathrm{fs}^{-2}$. Detuning from the resonance frequency is equal to $0.05\left(\omega_{0}=0.95 \omega_{\mathrm{QD}}\right)$.

\section{Conclusions}

The ability to generate a nanoconfined optical excitation at the end of a sharp probe tip with high nanofocusing efficiency holds significant promise for near-field control of nanostructures. Extension of ultrafast pulse manipulation to the nanoscale through plasmonic nanofocusing will allow for the all-optical control of the elementary excitation of matter on their characteristic time and length scales simultaneously. For a first-order process with number of quantum $n=1$, such as scattering, photoeffect, or fluorescence, an enhancement factor of three to four orders of magnitude is required. But for nonlinear processes of ionization and high-order harmonic generation, the required enhancement factor is near 50. SPP superfocusing can be used for spatially localized coherent control excitons in a semiconductor QD. In such experiments, short laser $\pi$-pulses are elected to coherently manipulate the exciton state.

Metallic nanocones are effective for the selective spatial excitation of quantum systems, in particular for QDs. A silver cone with parameters $\alpha=0.1$ is appropriate for excitation of a QD with a probability of $>0.98$ at the distances $y<6 \mathrm{~nm}$ and a probability of $<0.1$ at the distances $y>15 \mathrm{~nm}$. For high excitation selectivity, the initial parameters of the pulse should be accurately tuned.

\section{Acknowledgments}

The research was supported by the Russian Foundation for Basic Research (Grant Nos. 13-070027 and 015-07-09123 A) and by the government order of the RF Ministry of Education and Science (Project No. 1940).

\section{References}

1. R. S. Judson and H. Rabitz, "Teaching lasers to control molecules," Phys. Rev. Lett. 68, 1500-1503 (1992).

2. A. Assion et al., "Control of chemical reactions by feedback-optimized phase-shaped femtosecond laser pulses," Science 282, 919-922 (1998).

3. L. Novotny and B. Hecht, Principles of Nano-Optics, Cambridge University Press, Cambridge (2006).

4. M. W. Vogel and D. K. Gramotnev, "Adiabatic nano-focusing of plasmons by metallic tapered rods in the presence of dissipation," Phys. Lett. A 363(5-6), 507-511 (2007).

5. O. Keller, Quantum Theory of Near Field Electrodynamics, Springer, Heidelberg (2011).

6. S. A. Maier, Plasmonics: Fundamentals and Applications, Springer, New York (2007).

7. S. Berweger et al., "Light on the tip of needle: plasmonic nanofocusing for spectroscopy on the nanoscale," J. Phys. Chem. Lett. 3, 945-952 (2012).

8. M. I. Stockman, S. V. Faleev, and D. J. Bergman, "Coherent control of femtosecond energy localization in nanosystems," Phys. Rev. Lett. 88(6), 067402 (2002). 
9. M. Aeschlimann et al., "Adaptive subwavelength control of nano-optical fields," Nature 446, 301-304 (2007).

10. L. Cao et al., "Controlling plasmonic wave packets in silver nanovires," Nano Lett. 10(9), 3389-3394 (2010).

11. S. Berweger et al., "Femtosecond nanofocusing with full optical waveform control," Nano Lett. 11(10), 4309-4313 (2011).

12. A. V. Goncharenko, J. K. Wong, and Y. C. Chang, "Electric near-field enhancement of a sharp semi-infinite probe: material and cone angle dependence," Phys. Rev. B 74, 235442 (2006).

13. N. Issa and R. Guckenberger, "Optical nanofocusing on tapered metallic waveguides," Plasmonics 2, 31-37 (2007).

14. V. Kravtsov, J. M. Atkin, and M. B. Rashke, "Group delay and dispersion in adiabatic plasmonic nanofocusing," Opt. Lett. 38, 1322-1324 (2013).

15. F. De Angelis et al., "Multischeme approach for efficient surface plasmon polariton generation in metallic conical tips on AFM cantilevers," Opt. Express 19, 22268 (2011).

16. L. B. Felsen and N. Marcuvitz, Radiation and Scattering of Waves, Vol. 31, John Wiley \& Sons, New York (1994).

17. J. S. Lee et al., "Superfocusing of electric and magnetic fields using conical metal tips: effects of mod symmetry on the plasmon polariton method," Opt. Express 9, 12242 (2011).

18. A. J. Babajanyan, N. L. Margaryan, and K. V. Nerkararyan, "Superfocusing of surface polaritons in the conical structure," J. Appl. Phys. 87, 3785 (2000).

19. M. Agio, X. W. Chen, and V. Sandonghdar, "Nanofocusing radially-polarized beams for high-throughput funneling of optical energy to the near field," Opt. Express 18(10), 1087810887 (2010).

20. N. S. Koshlyakov, M. M. Smirnov, and E. B. Gliner, Differential Equations of Mathematical Physics, North-Holland Publishing Company, Amsterdam (1964).

21. A. D. Rakić et al., "Optical properties of metallic films for vertical-cavity optoelectronic devices," Appl. Opt. 37(22), 5271-5283 (1998).

22. B. Krummheuer, V. M. Axt, and T. Kuhn, "Theory of pure dephasing and the resulting absorption line shape in semiconductor quantum dots," Phys. Rev. B 65, 195313 (2002).

23. I. D'Amico and F. Rossi, "Field-induced Coulomb coupling in semiconductor macroatoms: application to single-electron quantum devices," Appl. Phys. Lett. 79, 1676 (2001).

24. L. Jacak, P. Hawrylak, and A. Wojs, Quantum Dots, Springer-Verlag, Heidelberg (1998).

25. A. Nazir et al., "Anticrossings in Förster coupled quantum dots," Phys. Rev. B 71, 045334 (2005).

26. M. G. Arustamyan and V. A. Astapenko, "Phase control of the excitation of a two-level system with short laser pulses," Laser Phys. 18(6), 768-773 (2008).

27. V. A. Astapenko and M. S. Romadanovskii, "Excitation of a two-level system by chirped laser pulse," Laser Phys. 19, 969-973 (2009).

28. V. Astapenko, Interaction of Ultrafast Electromagnetic Pulses with Matter, Springer, Heidelberg, New York, Dordrecht, London (2013).

Biographies for the authors are not available. 